\title{
Noise reduction on mammographic phantom images
}

\author{
M. Adel ${ }^{*}$, D. Zuwala ${ }^{+}$, M. Rasigni ${ }^{+}$and S. Bourennane ${ }^{*}$ \\ "Institut FRESNEL, UMR-CNRS 6133, Equipe GSM, Domaine Universitaire de Saint-Jérôme, 13397 Marseille Cedex \\ 20, France \\ ${ }^{+}$Interface Physique-Biologie-Médecine, case EC1, Domaine Universitaire de Saint-Jérôme, 13397 Marseille Cedex 20,
} France

Received 13 September 2005; accepted 27 October 2006

\begin{abstract}
A noise reduction scheme on digitized mammographic phantom images is presented. This algorithm is based on a direct contrast modification method using an optimal function which is obtained by means of the mean squared error as a criterion. Computer simulated images containing objects similar to those observed in the phantom are built to evaluate the performance of the algorithm. Noise reduction results obtained on both simulated and real phantom images show that the developed method may be considered as a good pre-processing step from the point of view of automating phantom film evaluation by means of image processing.
\end{abstract}

Key Words: noise reduction, signal-dependent noise, mammographic phantom, contrast modification.

\section{Introduction}

Mammography is the most effective and reliable method currently used for early breast cancer detection. Relevance of diagnosis mainly depends on mammographic image quality, among others are: adequate optical density range, good resolution and sharpness. It is the reason why periodic controls are made in mammographic facilities in order to ensure that everything in the mammographic chain works properly. In particular a mammographic phantom is used for this purpose. It is an object with the same radiological response (absorption properties) as an average dense breast, in which are embedded several targets simulating microcalcifications, masses or nodules and fibrous linear structures (fibres) of decreasing size and contrast. The mammographic phantom film is shown to several readers and a score is obtained by each of them, depending on the number of simulated objects they can see. The independent object visibility scores are then averaged and the resulting score is assigned to the phantom film. Automating this score by using computer image processing of digitized phantom film should make the evaluation of mammographic facilities easier and in particular less subjective.

In this framework a way for making detection and segmentation of features easier is to enhance them beforehand. Image enhancement is usually made by either suppressing noise or increasing image contrast. Several studies have been devoted to contrast enhancement on mammographic images, while few studies were concerned with noise reduction $[1,11,17,21]$.

Correspondence to: mouloud.adel@univ-cezanne.fr

Recommended for acceptance by $\mathrm{Dr}$. Weickert

ELCVIA ISSN: 1577-5097

Published by Computer Vision Center / Universitat Autonoma de Barcelona, Barcelona, Spain 
Techniques used for contrast enhancement on mammographic images include six broad categories: unsharp masking $[12,16]$, histogram equalization $[19,20]$, adaptive neighborhood contrast enhancement $[9$, $10,18,22]$, wavelet enhancement $[14,15]$, fuzzy modelling $[6,24]$ and spatial filtering $[5,25]$.

Among these techniques, adaptive contrast enhancement and wavelets proved to be more suitable for mammography [23]. Adaptive contrast enhancement methods use a local contrast definition and then image is enhanced by improving the contrast measure via a contrast modification function. Originally this method was proposed by Gordon and Rangayyan [10]. Dahwan et al. [9] noticed that the contrast enhancement functions used by Gordon and Rangayyan [10] introduced too much noise and they proposed alternative contrast modification functions such as the exponential and the logarithm ones. In their study [9] contrast measure was computed over an adaptive neighbourhood of variable shape and size. Another contrast measure based on edge information was defined by Beghdadi et al. [3]. Dash et al. [8] improved this method by modifying the contrast measure, introducing a lower degree of noise amplification and reducing the computational complexity. Chang et al. [4] also proposed an adaptive contrast enhancement technique based on a local standard deviation histogram transformation whereas Zhu et al. [27] used constrained local histogram equalization for enhancing details in an image while preserving its appearance. Recently Cheng et al. [7] presented a new homogeneity measure for contrast enhancement. Because of the very noisy nature of phantom images compared to mammographic images, in particular high frequency noise due to the phantom itself, the previous methods, widely used on mammographic images, are not directly usable for detecting embedded objects in the phantom in that they enhance both noise and signal.

In this letter, the performance of a noise reduction method based on a local contrast modification function is evaluated on computer simulated and real phantom images. First, a local contrast is computed for each pixel, depending on its neighbourhood statistical properties. An optimal contrast modification function to be determined is then applied. This function is found by solving an optimization problem using the mean squared error as a criterion. Finally the enhanced pixel value is calculated using an inverse local contrast method.

\section{Noise reduction scheme description}

Let I be an image of $\mathrm{N}_{\mathrm{x}} \mathrm{N}_{\mathrm{y}}$ pixels where each pixel $(\mathrm{i}, \mathrm{j})$ is coded into $\mathrm{n}_{\mathrm{g}}$ grey levels i.e. takes its value in the set $\left\{0,1 \ldots \mathrm{n}_{\mathrm{g}}-1\right\}$. The basic idea of the algorithm is to assign to each pixel $(\mathrm{i}, \mathrm{j})$, an inner and an external window centred on it, of $(2 p+1) \times(2 p+1)$ and $(2 p+3) \times(2 p+3)$ pixels respectively. Center area is defined as the set of pixels within the inner window whereas background area is the set of pixels between the inner and the external window. A local contrast image $\mathrm{C}$ is then defined as follows:

$$
\begin{aligned}
& C(i, j)=\frac{\left|M_{c}(i, j)-M_{b}(i, j)\right|}{\max \left[M_{c}(i, j), M_{b}(i, j)\right]}, \\
& \quad \text { with } \\
& C(i, j)=0 \quad \text { if } M_{c}(i, j)=M_{b}(i, j)=0,
\end{aligned}
$$

where $M_{c}(i, j)$ and $M_{b}(i, j)$ are the mean value of pixels belonging to the center and the background area respectively. Note that $\mathrm{C}(\mathrm{i}, \mathrm{j})$ is within the range [0,1]. A contrast modification function $\Psi$ whose determination is detailed in section 3, is then applied to image $\mathrm{C}$ in order to enhance features to be detected. The resulting image is denoted by $\mathrm{C}^{\prime}=\Psi(\mathrm{C})$. So $\Psi$ is defined as:

$$
\Psi:[0,1] \rightarrow[0,1]
$$

To transform image $C^{\prime}$ into the grey level domain of image $\mathrm{I}$, the following transform is applied: 


$$
\begin{array}{ll}
E(i, j)=\frac{M_{b}(i, j)}{1-C^{\prime}(i, j)} & \text { if } \quad M_{b}(i, j) \leq M_{c}(i, j) \quad, \quad \text { (a) } \\
E(i, j)=M_{b}(i, j)\left[1-C^{\prime}(i, j)\right] & \text { if } \quad M_{b}(i, j)>M_{c}(i, j) \quad \text { (b) }
\end{array}
$$

This transform yields an image E, which is an enhanced version of image I.

From a practical point of view, only inner and external window parts whose intersection with image I is not empty, are used for computing local contrast $C$ on image I boundary. In the case where $M_{b}(i, j) \leq M_{c}(i, j)$ and $C^{\prime}(i, j)=1$ we put $E(i, j)=n_{g}-1$.

\section{Optimal contrast modification function}

In the case of mammographic digitized films, noise due to film granularity and quantum mottle can be modeled as spatially correlated Poisson noise $[2,26]$. As a first order approximation a signal-dependent spatially uncorrelated Gaussian noise is used as follows [1,21]:

$$
I(i, j)=F(i, j)+W(i, j) \quad \text { with } \quad W(i, j)=\sqrt{F(i, j)} U(i, j),
$$

where $I(i, j)$ is the noisy observed image, $F(i, j)$ is the noise-free image, $W(i, j)$ is the signal-dependent noise and $\mathrm{U}(\mathrm{i}, \mathrm{j})$ is a zero-mean Gaussian noise with standard deviation $\sigma$.

First consider the case of computer simulated phantom images. With the objective of determining the function $\Psi$ leading to the best approximation $\mathrm{E}$ of noise-free image $\mathrm{F}$, we used the criterion consisting of minimizing the Mean Squared Error (MSE) between images F and E, defined as:

$$
\operatorname{MSE}=\frac{1}{N_{x} N_{y}} \sum_{(i, j) \in I}[F(i, j)-E(i, j)]^{2},
$$

where $\mathrm{N}_{\mathrm{x}} \mathrm{N}_{\mathrm{y}}$ is the total number of pixels in images $\mathrm{F}$, I and $\mathrm{E}$.

Let $\mathrm{H}$ and $\mathrm{K}$ be the two sets defined as:

$\mathrm{H}=\left\{(\mathrm{i}, \mathrm{j}) \in \mathrm{I} / \mathrm{M}_{\mathrm{b}}(\mathrm{i}, \mathrm{j}) \leq \mathrm{M}_{\mathrm{c}}(\mathrm{i}, \mathrm{j})\right\} \quad$ and $\quad \mathrm{L}=\left\{(\mathrm{i}, \mathrm{j}) \in \mathrm{I} / \mathrm{M}_{\mathrm{b}}(\mathrm{i}, \mathrm{j})>\mathrm{M}_{\mathrm{c}}(\mathrm{i}, \mathrm{j})\right\}$.

The function $\Psi$ is then approached as a piecewise constant function. The local contrast range $[0,1]$ is split up into M identical segments $h_{k}(k=1,2, \ldots . M)$. In each subinterval $h_{k}, \Psi$ is assumed to be a constant $\alpha_{k}$ :

$$
\Psi=\sum_{\mathrm{k}=1}^{\mathrm{M}} \alpha_{\mathrm{k}} 1_{-} \mathrm{h}_{\mathrm{k}}
$$

where $1 h_{k}$ stands for the indicator function of set $h_{k}, \alpha_{k}$ is the value taken by function $\psi$ in the subinterval $\mathrm{h}_{\mathrm{k}}$ and $\mathrm{h}_{\mathrm{k}}=\left[\frac{\mathrm{k}-1}{\mathrm{M}}, \frac{\mathrm{k}}{\mathrm{M}}\right]$.

By taking Eqs. 2 into account, Eq.4 becomes:

$$
\operatorname{MSE}=\frac{1}{N_{x} N_{y}} \sum_{k=1}^{M}\left[\sum_{(i, j) \in H_{k}}\left[F(i, j)-\frac{M_{b}(i, j)}{\beta_{k}}\right]^{2}+\sum_{(i, j) \in L_{k}}\left[F(i, j)-M_{b}(i, j) \beta_{k}\right]^{2}\right]
$$

where $\beta_{\mathrm{k}}=1-\alpha_{\mathrm{k}}, \quad \mathrm{H}_{\mathrm{k}}=\left\{(\mathrm{i}, \mathrm{j}) \in \mathrm{H} / \mathrm{C}(\mathrm{i}, \mathrm{j}) \in \mathrm{h}_{\mathrm{k}}\right\} \quad$ and $\quad \mathrm{L}_{\mathrm{k}}=\left\{(\mathrm{i}, \mathrm{j}) \in \mathrm{L} / \mathrm{C}(\mathrm{i}, \mathrm{j}) \in \mathrm{h}_{\mathrm{k}}\right\}$.

For a given $\mathrm{k}$ value, the condition $\frac{\partial \mathrm{MSE}}{\partial \beta_{\mathrm{k}}}=0$ yields: 


$$
\left(\sum_{(i, j) \in L_{k}} M_{b}(i, j)^{2}\right) \beta_{k}^{4}-\left(\sum_{(i, j) \in L_{k}} M_{b}(i, j) F(i, j)\right) \beta_{k}^{3}+\left(\sum_{(i, j) \in H_{k}} M_{b}(i, j) F(i, j)\right) \beta_{k}-\sum_{(i, j) \in H_{k}} M_{b}(i, j)^{2}=0
$$

So MSE minimization leads to solve the above equation for $\beta_{\mathrm{k}}$ for each segment $\mathrm{h}_{\mathrm{k}}$. This may be done on computer by means of algorithms devoted to root finding such as MATLAB software (algorithm used for that purpose involves computation of the eigenvalues of a companion matrix. These eigenvalues are the roots of the polynomial). Only $\beta_{\mathrm{k}}$ values less than one are taken into account. Thus the optimal contrast modification function $\Psi$ is defined by the set of $\alpha_{k}=1-\beta_{k}(k=1,2, \ldots M)$.

In the case of real phantom images the function is determined from results obtained on simulated phantom images as it is explained in the next section.

\section{$4 \quad$ Results and discussion}

First, in order to perform quantitative evaluation of the noise reduction method described in the previous sections, computer simulated images containing objects similar to those observed on the phantom were generated with different global contrast and noise levels using Eq. 3. Global contrast $\mathrm{C}_{\mathrm{G}}$ level is defined as

$$
\mathrm{C}_{\mathrm{G}}=\frac{\mathrm{G}_{\mathrm{o}}-\mathrm{G}_{\mathrm{b}}}{\mathrm{G}_{\mathrm{b}}}
$$

where $G_{o}$ and $G_{b}$ are the mean grey level of the object and the background respectively. Only poor contrast objects such as masses and fibres and in particular the largest in size mass and fibre are studied in this paper. For each previous target four noise-free images $\mathrm{F}$ corresponding to a global contrast level $\mathrm{C}_{\mathrm{G}}$ of $10,20,30$ and $40 \%$ were generated. Three signal-dependent noise W (Eq.3) were then associated with each noise-free image $\mathrm{F}$ for giving images I (Eq.3). Images W were generated so that image Signal-to-Noise Ratio (SNR) defined as

$$
\mathrm{SNR}_{\mathrm{dB}}=10 \log _{10}\left(\frac{\sum_{\mathrm{i}=1}^{N_{\mathrm{X}}} \sum_{\mathrm{j}=1}^{N_{\mathrm{y}}} \mathrm{F}(\mathrm{i}, \mathrm{j})^{2}}{\sum_{\mathrm{i}=1}^{\mathrm{N}_{\mathrm{x}}} \sum_{\mathrm{j}=1}^{N_{\mathrm{y}}} \mathrm{W}(\mathrm{i}, \mathrm{j})^{2}}\right)
$$

takes 21, 15 and 9dB values, which simulate low, intermediate and high noise levels respectively. Thus 12 images I were obtained for each target (mass or fibre). Images were coded on 256 grey levels and their sizes varied from 256x256 to 336x336 pixels for masses and fibres respectively. Figure 1 shows some of them.

For each image I, we varied the p parameter value characterizing the size of inner and external windows used for computing local contrast C (Eq.1). For each $p$ value we applied the method described in section 3 for determining the corresponding piecewise constant $\Psi$ function and hence by the way of Eqs. 2 and 4 we estimated the MSE. For a given image I the optimal $p$ value (optimal window size) was then defined as the one yielding the minimal MSE value among those previously computed. 


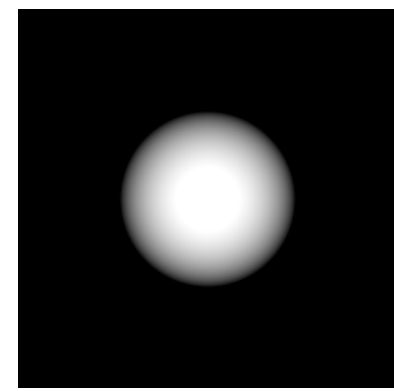

(a)

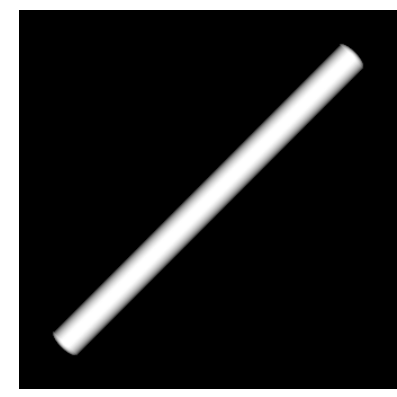

(e)

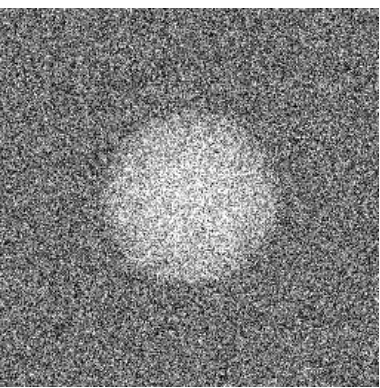

(b)

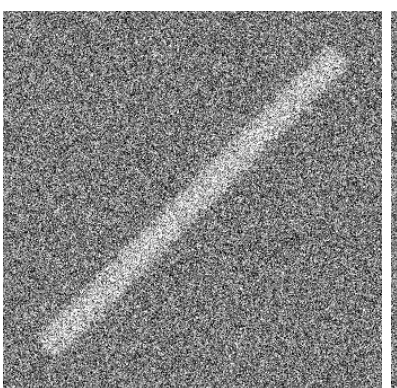

(f)

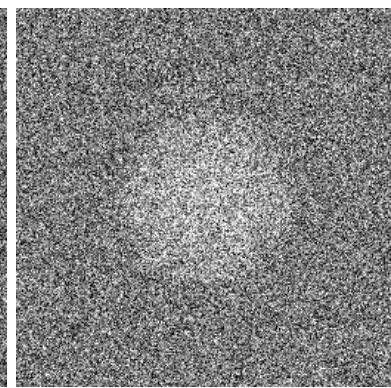

(c)

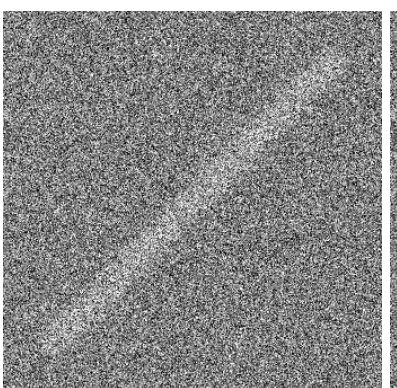

(g)

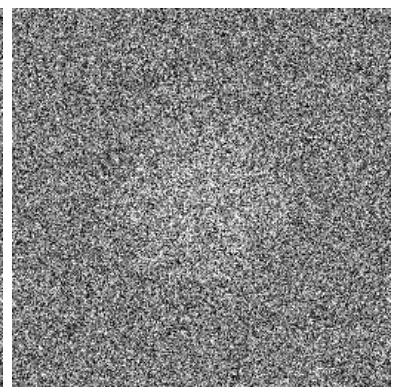

(d)

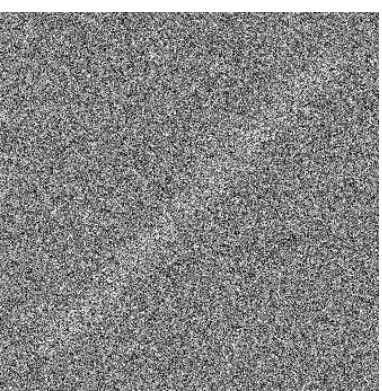

(h)

Fig. 1: Computer simulated images of a mass and a fibre

(a) and (e) mass and fibre noise-free images with a $\mathrm{C}_{\mathrm{G}}=10 \%$ global contrast level; (b), (c) and (d): noisy images with $\mathrm{SNR}=21 \mathrm{~dB}$ for (b), SNR $=15 \mathrm{~dB}$ for (c) and $\mathrm{SNR}=9 \mathrm{~dB}$ for (d); (f), (g) and (h) : same as (b) (c) and (d) for fibre in image (e).

Local contrast $\mathrm{C}$ values associated with optimal window sizes are relatively small and depend on noise level. They do not exceed 0.15 for highest noise level images. From a practical point of view $\Psi$ determination was made by using a subinterval length of $10^{-3}\left(\mathrm{M}=10^{3}\right)$. Figure 2 gives the plot of $\Psi$ versus local contrast $\mathrm{C}$ for masses of Figs. 1(b), 1(c) and 1(d). It may be seen that $\Psi$ varies linearly with $\mathrm{C}$ (more or less scattered $\Psi$ values for largest $\mathrm{C}$ are due to the fact that subintervals for these $\mathrm{C}$ values contain very few pixels yielding thus statistical fluctuations). Such a behaviour was observed for all computer simulated images. The solid lines in Fig.2 are least-square fits to the data and are described by equations $\Psi=a \mathrm{C}+b$, where $a$ and $b$ are 2 parameters. By taking the very small value of $b$ into account, $\Psi$ may be simply written in the form $\Psi=a \mathrm{C}$. Figure 3 shows images obtained after applying the noise reduction scheme with the corresponding optimal contrast modification function $\Psi=a \mathrm{C}$ on the masses and fibres of Fig. 1 (b,c,d,f,g,h). 


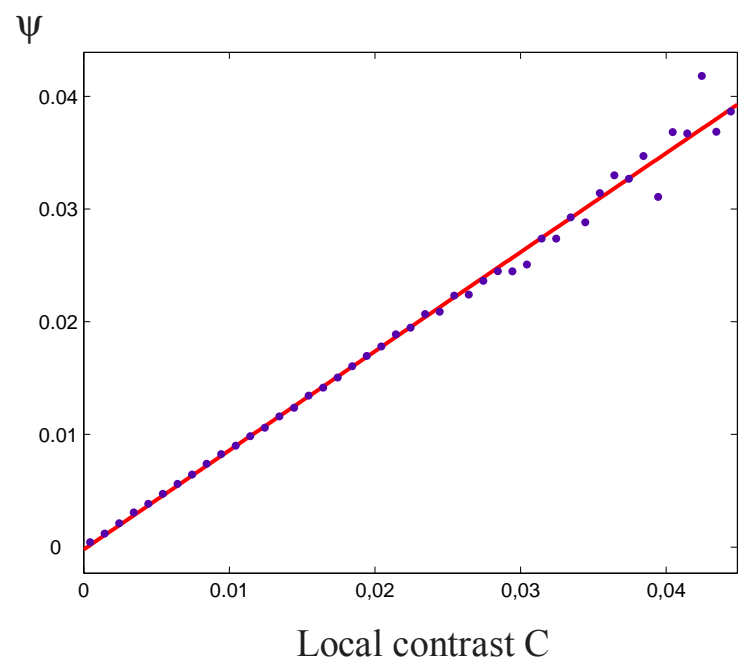

(a)

$\psi$

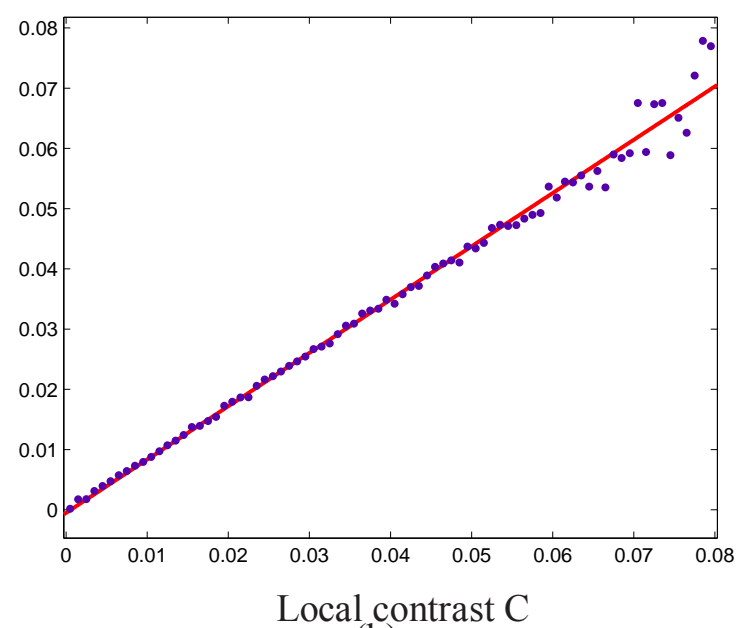

(b)

$\psi$

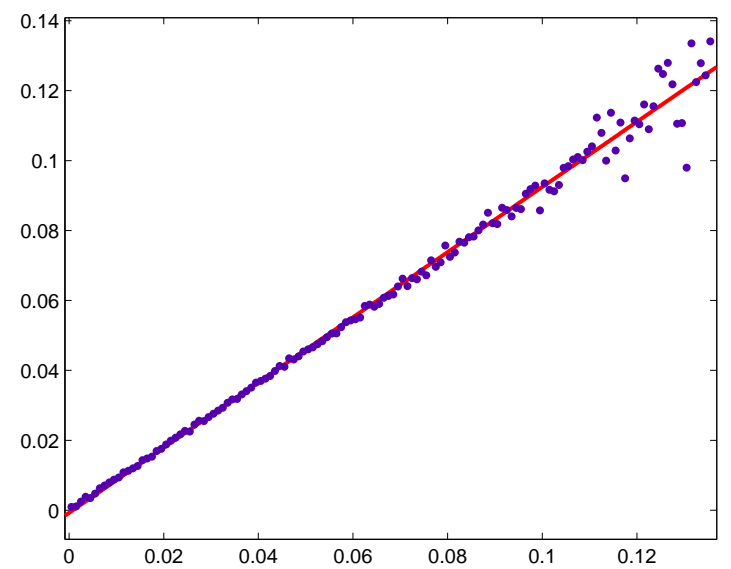

\section{Local contrast C}

(c)

Fig. 2: Optimal function $\Psi$ versus local contrast for :

(a) : image of Fig. 1(b); (b): image of Fig. 1(c); (c) : image of Fig. 1(d) 
By way of comparison median and adaptive noise smoothing filters (ANS) proposed by Kuan et. al. [13] were applied on simulated images. The window size used for each filter is the one giving minimal MSE value. Table 1 summarizes MSE values related to objects ( masses and fibres) of Fig. 1. As it can be seen, the proposed method yields the lowest MSE values.

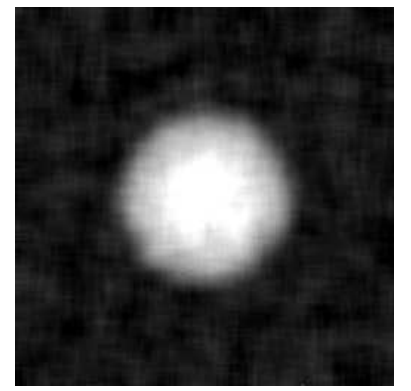

(a)

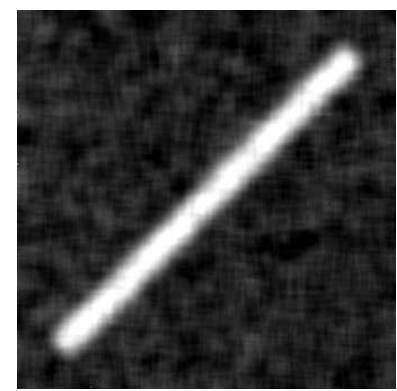

(d)

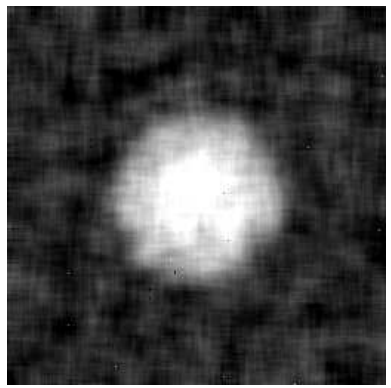

(b)

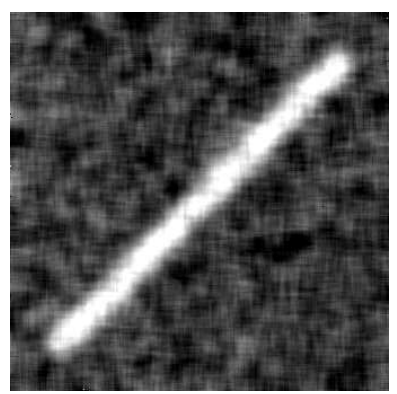

(e)

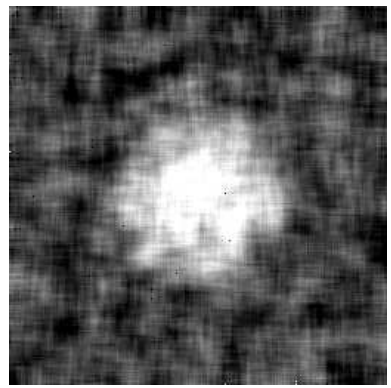

(c)

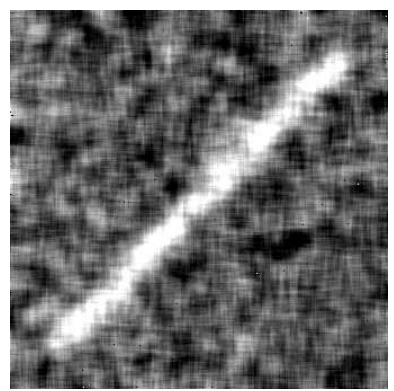

(f)

Fig. 3: Noise reduction results on computer simulated images for :

(a), (b) and (c): images of Fig. 1(b), 1(c) and 1(d) respectively.

(d), (e) and (f): images of Fig. 1(f), 1(g) and 1(h) respectively.

On the other hand tests on real images of phantom were also carried out. For that purpose 20 phantom films were digitized using a high drum scanner (Scanmate 11000, ScanView) with a spatial resolution of 50 $\mu \mathrm{m} /$ pixel, 8 bits/pixel. Two sub images(256x256 and 336x336 pixels) approximately centered on the largest mass and fibre respectively were extracted from each digitized phantom image. Global contrast $\mathrm{C}_{\mathrm{G}}$ and Signal-to-Noise ratio SNR were estimated for each sub image. These estimations enabled us to deduce from results on simulated images the corresponding $p$ and $a$ parameter values to be used in this case. Figure 4 shows results obtained from two digitized phantom images. As for simulated images, targets appear more visible after treatment. 


\begin{tabular}{|c|c|c|c|c|c|c|}
\cline { 2 - 7 } & \multicolumn{6}{c|}{ MSE parameter } \\
\cline { 2 - 7 } & \multicolumn{3}{c|}{ Mass images } & $1(\mathrm{f})$ & $1(\mathrm{~g})$ & $1(\mathrm{~h})$ \\
\cline { 2 - 7 } & $1(\mathrm{~b})$ & $1(\mathrm{c})$ & $1(\mathrm{~d})$ & 137 & 556 & 2188 \\
\hline $\begin{array}{c}\text { Noisy } \\
\text { image }\end{array}$ & 140 & 561 & 2237 & 2.04 & 4.19 & 8.32 \\
\hline $\begin{array}{c}\text { Median } \\
\text { filter }\end{array}$ & 0.99 & 2.38 & 4.83 & 2.83 & 5.30 & 7.92 \\
\hline $\begin{array}{c}\text { ANS Filter } \\
\text { Proposed } \\
\text { method }\end{array}$ & 1.68 & 2.84 & 5.01 & $\mathbf{1 . 4 6}$ & $\mathbf{2 . 9 1}$ & $\mathbf{5 . 3 9}$ \\
\hline
\end{tabular}

Table 1: MSE parameter ( on the object) evaluated for objects (masses and fibres) on images shown in figs. 1 (b), (c), (d), (f), (g), (h) after applying the proposed method, the median filter and the Adaptive Noise Smoothing filter (ANS) according to (Kuan et al. 1985). MSE parameter is also evaluated for each noisy image before processing.

\section{Conclusion}

In this paper we described a noise reduction method on digitized phantom images using an optimal contrast modification function. Such a function was obtained from computer simulated phantom images by considering the mean squared error as a criterion. Noise reduction scheme turned out to provide good results both in simulated and real phantom images. So it might be the first step of a feasibility study for automating quantitative phantom image analysis by means of image processing techniques. 


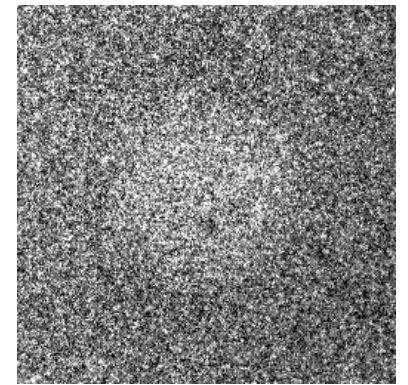

(a)

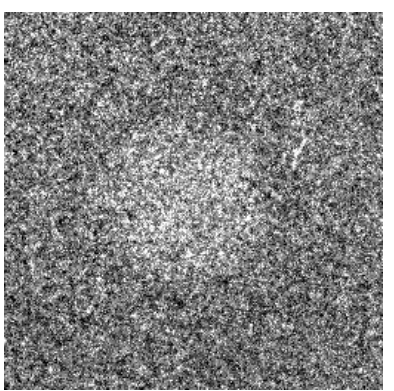

(c)

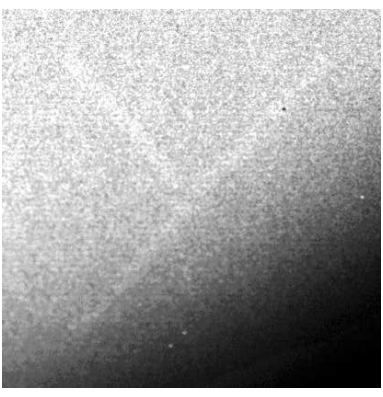

(e)

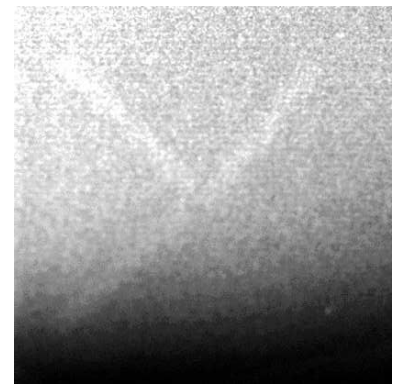

(g)

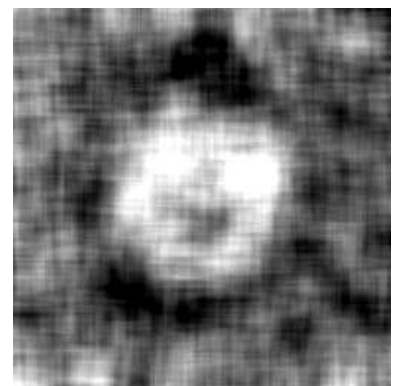

(b)

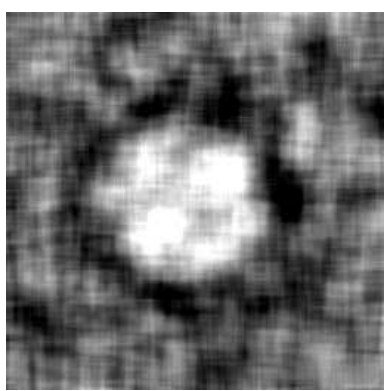

(d)

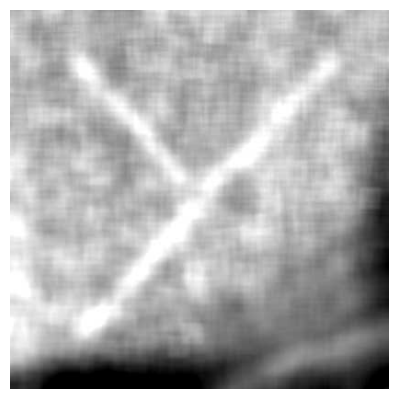

(f)

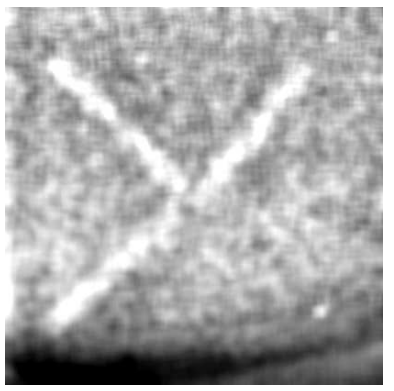

(h)

Fig. 4: Noise reduction on real images of phantom

(a) and (c) original mass images; (b) and (d) resulting images after treatment. (e) an (g) original fibre images; (f) and (h) resulting images after treatment. 


\section{References}

[1] F. Aghadasi, R.K. Ward, B. Palcic, "Noise filtering for mammographic images", Proceeding of the $14^{\text {th }}$ Annual International Conference of the I.E.E.E. Engineering in Medecine and Biology Society, 1877-1878, 1992.

[2] G.T. Barnes, D.P. Chakraborty, "Radiographic mottle and patient exposure in mammography", Radiology, 145:815-821, 1982.

[3] A. Beghdadi, A. Le Negrate, "Contrast Enhancement Technique Based on Local Detection of Edges”, Comput. Vision, Graphics, Image Processing, 46:162-174, 1989.

[4] D. Chang, W. Wu, "Image contrast enhancement based on a histogram transformation of local standard deviation", IEEE Trans. Med. Imaging, 17(4):518-531, 1998.

[5] H. Chen, A. Li, L. Kaufman, J. Hale, "A fast filtering algorithm for image enhancement”, IEEE Trans. Med. Imaging, 13(3):557-564, 1994.

[6] H.D. Cheng, H. Xu, "A novel fuzzy logic approach to mammogram contrast enhancement", Information Sciences, 148:167-184, 2002.

[7] H.D. Cheng, M. Xue, X.J. Shi, "Contrast enhancement based on a novel homogeneity measurement", Pattern Recognition, 36(11):2687-2697, 2003.

[8] L. Dash, B.N. Chatterji, "Adaptive contrast enhancement and de-enhancement", Pattern Recognition, 24(4):289-302, 1991.

[9] A.P. Dhawan, G. Buelloni, R. Gordon, "Enhancement of mammographic features by optimal adaptative neighborhood image processing", IEEE Trans. Med. Imaging, 5(1):8-15, 1986.

[10] R. Gordon, R.M. Rangayyan, "Feature enhancement of film mammograms using fixed and adaptative neighbourhoods", Applied Optics, 23(4):560-564, 1984.

[11] V. Guis, M. Adel, M. Rasigni, G. Rasigni, B. Seradour, P. Heid, "Adaptive neighborhood contrast enhancement in mammographic phantom images", Opt. Eng, 42(2):357-366, 2003.

[12] J.K. Kim, J.M. Park, K.S. Song, H.W. Park, "Adaptive mammographic image enhancement using first derivative and local statistics", IEEE Trans. Med. Imaging, 16(5): 495-502, 1997.

[13] D.T. Kuan, A.A Shawchuk, T.C. Strand, P. Chavel, "Adaptive noise smoothing filter for images with signal-dependent noise", IEEE Trans. Pattern Anal. Mach. Intell, 7(2):165-177, 1985.

[14] A.F. Laine, S. Schuler, J. Fan, W. Huda, "Mammographic feature enhancement by multiscale analysis", IEEE Trans. Med. Imaging 13(4), 725-740, 1994.

[15] A.F. Laine, J. Fan, W. Yang, "Wavelets for contrast enhancement of digital mammography", IEEE Eng. Med. Biol. Magazine, 14:536-550, 1995.

[16] F.Y.M. Lure, P.W. Jones, R.S. Gaborski, "Multi-resolution unsharp masking technique for mammogram image enhancement", Proc. SPIE 2710, 830-839, 1996.

[17] J. Martens, "Adaptive contrast enhancement through residue-image processing", Signal Processing, 44:1-18, 1995.

[18] W.M. Morrow, R.B.Paranjape, R.M. Rangayyan, "Region based contrast enhancement of mammograms", IEEE Trans. Med. Imaging, 11(3):392-406, 1992.

[19] R.B. Paranjape, W.M. Morrow, R.M. Rangayyan, "Adaptive neighborhood histogram equalization for image enhancement", Comput. Vision, Graphics, Image Processing, 54:259267, 1992.

[20] R.M. Pizer, E.P. Amburn, J.D. Austin, R. Cromartie, A. Geselowitz, T. Greer, B. Ter Haar Romeny, J.B. Zimmerman, K. Zuiderveld, "Adaptive histogram equalisation and its variations", Comput. Vision, Graphics, Image Processing, 39:355-368, 1987. 
[21] W. Qian, L.P. Clarke, M. Kallergi, R.A. Clark, "Tree-Structured Nonlinear Filters in Digital Mammography", IEEE Trans. Med. Imaging, 13(1):25-36, 1994.

[22] R.M. Rangayyan, L. Shen, Y. Shen, J.E.L. Desautels, H. Bryant, T.J. Terry, N. Horeczko, M.S. Rose, "Improvement of sensivity of breast cancer diagnosis with adaptive contrast enhancement", IEEE Trans. Inform. Technol. Biomed, 1:161-170, 1997.

[23] R. Sivaramakrishna, N.A. Obuchowski, W.A. Chilcote, G. Cardenosa, K. A. Powell, "Comparing the performance of mammographic enhancement : a preference study", American Journal of Radiology, 175:45-51, 2000.

[24] M.A. Sutton, J. Bezdek, "Enhancement and analysis of digital mammograms using fuzzy models", Proc. SPIE 3240,179-190, 1997.

[25] P.G. Tahoces, J. Correa, M. Souto, C. Gonzales, L. Gomez, J.J.Vidal, "Enhancement of chest and breast radiographs by automatic spatial filtering", IEEE Trans. Med. Imaging, 10(3):330$335,1991$.

[26] Steve Webb, The Physics of Medical Imaging, IOP Publishing, Adam Hilger, Bristol and Philadelphia, 1988.

[27] H. Zhu, F.H.Y. Chan, F.K. Lam, "Image contrast enhancement by constrained local histogram equalization”, Comput.Vision, Image, Understanding, 73(2):281-290, 1999. 